\title{
Presencia, abundancia y estrategias reproductivas de helechos en áreas alteradas de la Sierra Nevada, México
}

\author{
Ma. Lucía Rodríguez Romero ${ }^{1}$, José Alejandro Zavala Hurtado ${ }^{2} \&$ Leticia Pacheco $^{3}$ \\ 1. Doctorado en Ciencias Biológicas, Depto. de Biología, Universidad Autónoma Metropolitana-Iztapalapa, Apdo. \\ Postal 55-535, 09340 México, D.F., México; rrlucia@hotmail.com \\ 2. Área de Ecología, Depto. de Biología, Universidad Autónoma Metropolitana-Iztapalapa, Apdo. Postal 55-535, 09340 \\ México, D.F., México; jazh@xanum.uam.mx \\ 3. Área de Botánica Estructural y Sistemática Vegetal, Depto. de Biología, Universidad Autónoma Metropolitana- \\ Iztapalapa, Apdo. Postal 55-535, 09340 México, D.F., México; pacheco@xanum.uam.mx
}

Recibido 16-II-2010. Corregido 15-VIII-2010. Aceptado 13-IX-2010.

\begin{abstract}
Presence, abundance and reproductive strategies of ferns in disturbed areas of Sierra Nevada, México. Diverse reproductive strategies shown by ferns and lycophytes allow them to colonize a variety of habitats, particularly after the incidence of natural or anthropogenic disturbances. This study assessed the presence, abundance and reproductive strategies of ferns growing in soils of temperate forests with different levels of disturbance at the Sierra Nevada mountain range in the State of Mexico. Vegetation, soil and environmental variables were recorded in $200 \mathrm{~m}^{2}$ permanent plots located in four forest stands. One-Way ANOVA and Canonical Correspondence Analysis resulted in the recognition of three landscape types defined by the degree of environmental alteration: low, moderate and severe. Also, from five soil samples collected in each stand, germination of ferns and lycophytes was induced. A positive relationship was found between the alteration degree and Cheilanthes abundance. Under a low landscape alteration regime, species richness is restricted to Cheilanthes bonariensis, C. marginata and Pellaea ternifolia subsp. ternifolia. The soil is a reservoir of spores of Cheilanthes and Pellaea ternifolia subsp. ternifolia because their spores can remain viable for different time intervals. Apogamy is the usual reproductive strategy of Cheilanthes species in disturbed ecosystems, although these species also show sexual reproduction in natural ecosystems with adequate water availability. Apogamy may be related to a shorter generation time in comparison with a low disturbed ecosystem. On the other hand, Pellaea ternifolia subsp. ternifolia only has sexual reproduction. Apogamy might be related to a faster generation in comparison with a low disturbed ecosystem. Rev. Biol. Trop. 59 (1): 417-433. Epub 2011 March 01.
\end{abstract}

Key words: apogamy, environmental alteration, ferns, lycophytes, Mexico, soil spore bank.

El peculiar ciclo biológico y los diversos mecanismos y estrategias reproductivas que tienen los helechos (Lloyd 1974, Bell 1979, Smith 1979, White 1979, Sheffield \& Bell 1987, Farrar et al. 2008, Sheffield 2008), favorecen su capacidad de invadir ambientes perturbados como laderas taladas y bosques recientemente quemados. Esto explica su presencia en las primeras etapas de sucesión y su éxito colonizando espacios vacíos adecuados, que se abren de manera irregular y que son de duración limitada (Dyer \& Lindsay 1992,
Rodríguez et al. 2008). Además, se reproducen en un tiempo relativamente corto y se pueden conservar poblaciones in vivo en condiciones de laboratorio.

Los helechos y licófitas, como parte del sotobosque, comparten espacio físico con las fanerógamas herbáceas. Rincón-Gallardo (1991) menciona que no se ha determinado claramente su papel ecológico; sin embargo, para Page (1979), los helechos tienen relevancia en diferentes tipos de vegetación debida, en parte, a la diversidad de formas de vida. 
Se encuentran en todas las regiones, excepto en las extremadamente áridas y tienen un alto grado de variación genética asociado a una gran capacidad de dispersión y potencial de autofecundarse (Tryon 1986).

Las esporas de los helechos y licófitas son fácilmente dispersadas por el viento a grandes distancias, no obstante el patrón de distribución depende de la disponibilidad de un hábitat conveniente, variabilidad genética y estrategias reproductivas de las diferentes especies. Wherry (1920) estudió las diferencias entre presencia y abundancia de ciertas especies de helechos, correlacionando parámetros edáficos como pH y contenido de calcio. Kruckeberg (1964) los relacionó con suelos derivados de rocas de silicatos de hierro y magnesio, y Valdés \& Flores (1983) los estudiaron en suelos yesosos o salinos. Por otro lado Tuomisto \& Ruokolainen (1994), Tuomisto \& Poulsen (1996), Tuomisto et al. (1998) los vincularon a la textura y contenido de materia orgánica. El estudio de Rodríguez et al. (2008) relaciona la distribución de los helechos con características edáficas y ambientales e intuyen que la apogamia puede estar relacionada con el tipo de suelo.

El objetivo de este trabajo fue describir la presencia, abundancia y estrategias reproductivas de helechos y licófitas que crecen en diferentes tipos de suelo de bosques alterados de clima templado, en la Sierra Nevada, Municipio de Texcoco, Estado de México, con la suposición de que la apogamia está relacionada con el suelo alterado por la remoción de la vegetación y la entrada de mayor cantidad de luz.

\section{MATERIALES Y MÉTODOS}

Área de estudio: El trabajo se realizó en la Sierra Nevada, municipio de Texcoco, estado de México. El sistema orográfico de la Sierra, en Texcoco, se conforma de 29 elevaciones importantes, que van de los 2500 hasta $4100 \mathrm{~m}$ y pertenece a la provincia fisiográfica del Eje Neovolcánico y a la subprovincia Lagos y Volcanes de Anáhuac (INEGI 2000, Cosío 2001). El clima es semi-frío subhúmedo con lluvias en verano, de mayor humedad $\mathrm{C}(\mathrm{E})(\mathrm{w} 2)$, templado subhúmedo con lluvias en verano, de mayor humedad $\mathrm{C}(\mathrm{w} 2)$ y $\mathrm{C}(\mathrm{w} 1)$ templado subhúmedo con lluvias en verano, de humedad media (García \& Köeppen 1973). En el intervalo de 2600 a $3000 \mathrm{~m}$ de altitud, la precipitación anual es de 800 a 1 100mm (Cosío 2001). El tipo de suelo predominante es andosol y en menor proporción cambisol. La franja erosionada comprende montes y cañadas situados entre la Sierra Tláloc y el cerro de Tlaixpan (INEGI 2000, Cosío 2001). La vegetación consiste de bosques templados conformados por Quercus, Pinus y Abies que se ubican en la Sierra Quetzaltépetl y el Parque Nacional Zoquiapan (INEGI 2000).

Se realizó un recorrido prospectivo en la Sierra para identificar paisajes y alteraciones o perturbaciones evidentes, se ubicaron rasgos reconocibles como veredas rurales, cruces de camino, formas geográficas sobresalientes y tipos de vegetación. A partir de lo anterior, se seleccionaron cuatro zonas de estudio tomando en cuenta tipo de vegetación, cobertura y densidad vegetal, además del grado de erosión, evidenciado por la formación y tamaño de cárcavas. La caracterización de las áreas se apoyó utilizando las cartas temáticas E14B31 del Instituto Nacional de Estadística, Geografía e Informática (INEGI) a escala 1:50000. Además de obtener datos generales de longitud, latitud y altitud con un geoposicionador 12XL Garmin, versión 2.01 .

Zona A. Tequexquinahuac, ubicado a $19^{\circ} 28^{\prime} 08^{\prime \prime} \mathrm{N}, 98^{\circ} 49^{\prime} 03^{\prime \prime} \mathrm{W}$ y una altura promedio de $2512 \mathrm{msnm}$. La vegetación primaria es un bosque de Quercus con plantaciones cultivadas de pino y eucalipto. El suelo es somero y delgado con pequeñas porciones erosionadas.

Zona B. San Pablo Ixáyoc, se localiza entre los cerros Tepechichilco y Cuacale, con altitud que fluctúa entre 2560 a $2650 \mathrm{msnm}$ y está ubicado a $19^{\circ} 28^{\prime} 03^{\prime \prime} \mathrm{N}, 98^{\circ} 47^{\prime} 06^{\prime \prime} \mathrm{W}$. La zona se forma por pequeños parches de vegetación de Quercus, Abies y Pinus con evidencia de tala de árboles e incendios. El suelo es 
profundo, formado por aluviales de textura fina y las pendientes son pronunciadas.

Zona C. Tláloc, se localiza entre las cañadas Atlapulco y Carcases en las faldas del Tláloc, a $19^{\circ} 26^{\prime} 10^{\prime \prime} \mathrm{N}, 98^{\circ} 45^{\prime} 49^{\prime \prime} \mathrm{W}$, entre 2 820 a $3000 \mathrm{msnm}$. La vegetación consiste de Abies y Quercus. El suelo es profundo, las pendientes son pronunciadas y la alteración al ecosistema no es evidente.

Zona D. Santa Catarina del Monte, ubicada a $19^{\circ} 29^{\prime} 09^{\prime \prime} \mathrm{N}$ y $98^{\circ} 46^{\prime} 10^{\prime \prime} \mathrm{W}$, a una altura que oscila entre 2625 a $2671 \mathrm{msnm}$. La vegetación está constituida por relictos de bosque de Quercus y parches sin vegetación formados por grandes cárcavas, producto de erosión severa.

En cada área seleccionada se marcó una parcela permanente de $200 \mathrm{~m}^{2}$; excepto en la Zona A, donde se instalaron dos cuadrantes permanentes de $100 \mathrm{~m}^{2}$; uno en un relicto de bosque de encino y el segundo en una plantación de pino. En cada parcela se establecieron cinco subunidades de muestreo de un metro cuadrado cada una, cuatro de ellas en los vértices del cuadrante y una en el centro. En cada subunidad de muestreo se registraron características edáficas básicas como la textura por tacto (Siebe et al. 1996), tipo de sustrato y materia orgánica. Se estimaron parámetros como altitud, pendiente, exposición y tipo de vegetación (Rzedowski 1978). El nivel de alteración se determinó visualmente considerando tipo de vegetación, cultivos o senderos cercanos, evidencias de incendio, desmonte y erosión (Apéndice A).

De cada subunidad se extrajeron muestras de suelo, eliminando previamente el mantillo, de aproximadamente $500 \mathrm{~g}$ a una profundidad de $10 \mathrm{~cm}$; las muestras se depositaron en bolsas de polietileno negro para su transporte al laboratorio. El pH se midió en una suspensión de suelo y agua 1:25 con un potenciómetro digital Hanna Instruments HI 8314, el valor de pH se comparó con el cuadro propuesto por Siebe et al. (1996). El porcentaje de materia orgánica se determinó con la técnica desarrollada por Walkley \& Black (1934) que consiste en la oxidación de materia orgánica utilizando una reacción con dicromato de potasio y ácido sulfúrico; el resultado se valoró con la clave propuesta por Siebe et al. (1996). La determinación de color del suelo en seco y húmedo se llevó a cabo utilizando las cartas de Munsell (Department of Agriculture 1998).

Helechos y licófitas: Se recolectaron ejemplares de helechos y licófitas terrestres conforme al método clásico (Lorea \& Riba 1990). Al mismo tiempo, se anotó la presencia de éstos en cada parcela. Se determinaron con base en la obra de Mickel \& Smith (2004). El material de respaldo se depositó en el Herbario Metropolitano "Dr. Ramón Riba y Nava Esparza" (UAMIZ). Cuando se observaron gametófitos en campo, éstos se llevaron al laboratorio en donde se observó su desarrollo y estrategias reproductivas hasta la fase de esporófito.

Banco de esporas del suelo: Cada una de las muestras de suelo, se tamizaron en el laboratorio con una malla de acero de $0.84 \mathrm{~mm}$ de abertura para separar gravas y restos vegetales, con el fin de depositar $75 \mathrm{~g}$ de suelo tamizado en vasos térmicos desechables de $120 \mathrm{ml}$ adicionándoles agua suficiente para inducir la germinación. Se cubrieron con vasos de plástico transparente y se regaron cada semana con agua potable hasta obtener esporófitos. Se revisaron periódicamente con el fin de contar los gametófitos y esporófitos en cada recipiente y se anotó la presencia de anteridios y arquegonios hasta que se dio la fase esporofítica. Se consideraron esporófitos de origen apogámico cuando el gametófito formó un esporófito sin fusión sexual, es decir, no se observó presencia ni de anteridios ni arquegonios en todo el cultivo.

Los cultivos se mantuvieron en condiciones de laboratorio con luz artificial (lámparas de luz blanca de 20watts), con un fotoperiodo de 12 horas luz-oscuridad y temperatura de $20-27^{\circ} \mathrm{C}$, el medio fue humedecido cada semana con agua potable.

Se construyó una matriz de variables físicas y edáficas y otra que corresponde al número 
máximo de gametófitos y esporófitos, además del número de individuos por especie obtenidos al inducir germinación en 20 muestras de suelo. La evaluación estadística de germinación de esporas y la comparación del número de esporófitos de origen apogámico entre áreas de estudio, se llevó a cabo con un análisis de varianza no paramétrico de Kruskal-Wallis (Spiegel \& Stephens 2002), con un nivel de significancia de 0.95 .

La comparación de la velocidad de germinación entre sitios de estudio se realizó considerando el número de gametófitos a los 21 y 50 días después de inducir su germinación y se evaluó mediante un análisis de varianza de dos vías. También se evaluó el cambio de generación de gametófito a esporófito en las esporas germinadas, es decir el número de gametófitos de 50 días y de esporófitos de hasta 65 días después de inducir germinación entre áreas de estudio, a través de un análisis de varianza de dos vías (Spiegel \& Stephens 2002). Los análisis estadísticos se realizaron con un nivel de significancia de 0.95 .

Con el fin de analizar los patrones de distribución y abundancia de las 32 especies en las 20 muestras y su relación con las variables medioambientales, se realizó un análisis multivariado de correlación canónica (Kovach 2004). De esta manera se pudo evaluar la importancia de cada una de las variables en la determinación de los patrones espaciales de las comunidades. El análisis se realizó con el paquete MVSP, versión 3.12 (Kovach 2004).

\section{RESULTADOS}

Se observó la existencia de tres zonas con características diferentes: Tláloc, San Pablo Ixáyoc y Tequexquinahuac, Santa Catarina del Monte. La primera consistió en vegetación primaria o bosques cultivados, cobertura densa y continua, suelos profundos de color pardo oscuro o amarillos y de textura franco arcillosa, $\mathrm{pH}$ de moderadamente ácido a ligeramente alcalino (5.58-7.64), alto contenido de materia orgánica con excepción del suelo arenoso y con baja alteración evidente, sobre todo en el Tláloc.

La segunda zona en San Pablo Ixáyoc y Tequexquinahuac, tuvo pequeños parches de vegetación primaria de Quercus suelos generalmente amarillos, moderadamente profundos, la textura del suelo fue diversa, desde franco arenosa a franco arcilloso, el pH osciló de moderado a ligeramente ácido (5.52-6.24), el contenido de materia orgánica fue medio o medio alto, la alteración evidente fue alta en Tequexquinahuac y Santa Catarina del Monte, ya que los parches de vegetación primaria estaban rodeados por áreas erosionadas; en cambio, en San Pablo Ixáyoc la alteración se evidenciaba por parches con pruebas evidentes de incendios.

La tercera zona en Santa Catarina del Monte, se caracterizó por: alta degradación a lo largo de una amplia franja erosionada, algunas veces con profundas cárcavas; cobertura vegetal escasa o inexistente; los suelos delgados, someros e incluso sin horizonte A, cubiertos de hojarasca acarreada por el viento; $\mathrm{pH}$ entre 5.18 y 6.68; predominio de suelos de color pardo claro, textura compuesta principalmente por arcillas y grado de alteración muy alto, característico de la Zona D.

Helechos y Licófitas: Se determinó un total de 31 especies de helechos y licófitas distribuidas en 15 géneros y 7 familias (Apéndice B). La familia con mayor número de especies fue Pteridaceae, seguido de Polypodiaceae y Woodsiaceae. El género con mayor frecuencia de especies y de individuos fue Cheilanthes con excepción de la Zona $\mathrm{C}$ en donde se encuentra únicamente $C$. lendigera, destacando por su presencia $C$. bonariensis y $C$. marginata, sobre todo en las áreas más alteradas.

Asplenium, Pleopeltis y Polypodium son exclusivos de áreas con baja alteración evidente, Athyrium y Dryopteris sólo se observaron en áreas con muy baja alteración al igual que Cheilanthes lendigera, el género Selaginella sólo fue posible observarlo en la Zona A (Apéndice B). Es necesario subrayar que en 
lugares en donde hubieron incendios, se da una abundancia de Cheilanthes marginata.

Las muestras de gametófitos silvestres correspondieron a Adiantum poiretti, Asplenium monanthes, Cheilanthes bonariensis y Pellaea ternifolia subsp. ternifolia y se recolectaron en las Zonas B y C, en la parte media de Tepechichilco y en la parte baja de la cañada Atlapulco, su desarrollo fue acelerado en comparación con los que germinaron en condiciones de laboratorio y pese a la presencia de algas, fueron resistentes a la competencia.

Cuando se recolectaron los gametófitos silvestres se observaron anteridios y arquegonios y una semana después las primeras hojas del esporófito, sin embargo, éstos no formaron esporangios después de tres años de estudio en Cheilanthes bonariensis y Pellaea ternifolia subsp. ternifolia, a diferencia de Adiantum poiretti, el cual presentó esporangios y esporas al final de los tres años. Los esporófitos de mayor talla (más de $15 \mathrm{~cm}$ de altura) fueron $C$. bonariensis y P. ternifolia subsp. ternifolia.

Banco de esporas del suelo: La germinación y el desarrollo de gametófitos se encontró en 19 de las 20 muestras de suelo en las que se propició la germinación de esporas; la excepción fue la muestra C3 que correspondió a la Zona C. Los primeros esporófitos fueron de la muestra de suelo B4, que se recolectó en un parche en donde hubo evidencias de incendio (Zona B), estos esporófitos fueron de origen apogámico y se observaron 50 días después de haber inducido su germinación (Cuadro 1).

La fase esporofítica inició entre 51 y 65 días en 14 muestras, pero los gametófitos que les dieron origen no desarrollaron gametangios, por lo tanto fueron considerados apogámicos. A los 65 días, en algunos gametófitos surgieron anteridios y arquegonios y después de 100 días de haber inducido la germinación se observaron esporófitos, probablemente de origen sexual (Cuadro 1). Una situación diferente se observó en la muestra C2, en donde germinaron ocho individuos que permanecieron como gametófitos hasta después de un año y sólo des- pués de 900 días, seis individuos completaron su ciclo, es decir, formaron esporangios.

En las 19 muestras de suelo germinaron 1 538 individuos, de estos 962 formaron esporófitos con 862 de origen apogámico. Los esporófitos encontrados en las muestras A1, $\mathrm{B} 2, \mathrm{C} 1, \mathrm{C} 2, \mathrm{C} 3$ y C4 fueron de origen sexual, sin embargo, las muestras B4 y D1 suman 389 esporófitos apogámicos. Destaca la Zona C con el menor número de gametófitos (51) y un total de 18 esporófitos con la mitad de éstos apogámicos. En contraste la Zona D tuvo 895 gametófitos y 630 esporófitos de los cuales 421 fueron apogámicos.

Del total de gametófitos registrados en las 19 muestras, sólo 93 individuos (6.05\%) sobrevivieron y completaron su ciclo al formar esporangios. El conjunto de muestras de la Zona A sumaron 31 individuos que sobrevivieron hasta formar esporas (Apéndice C), destacando Cheilanthes bonariensis con 24 individuos. En las Zonas B y D, C. marginata tuvo una mayor frecuencia con 13 y 15 individuos respectivamente mientras que en la Zona $\mathrm{C}$ la mayor frecuencia fue para $C$. bonariensis y Pellaea ternifolia subsp. ternifolia con seis individuos cada una.

Por otro lado, en las cuatro áreas de trabajo se observaron 31 especies distintas de helechos y licófitas, pero en la muestra de suelo $\mathrm{C} 4$ se desarrolló la especie Astrolepis integerrima, la cual no había sido recolectada durante los recorridos previos (Cuadro 1).

En resumen, del total de individuos que completaron su ciclo de vida en el banco de esporas de suelo fueron: Cheilanthes bonariensis $45.16 \%$, C. marginata $31.18 \%$ y Pellaea ternifolia subsp. ternifolia $11 \%$.

El número de gametófitos de 21 días fue significativamente diferente entre las áreas de estudio (Cuadro 2). En el Cuadro 3 se muestra el resultado del análisis de varianza no paramétrico que indicó que si existe diferencia significativa $(\mathrm{p}=0.022)$ entre las áreas con el número de esporófitos de origen apogámico.

La variación resultó ser significativa entre áreas (Cuadro 4) al igual que en la germinación debido al número de días $(\mathrm{p}<0.05)$. La 
CUADRO 1

Presencia de gametófitos y esporófitos en el banco de esporas del suelo

TABLE 1

Presence of gametophytes and sporophytes in spore bank in soil

\begin{tabular}{|c|c|c|c|c|c|c|c|c|c|c|c|c|c|c|c|}
\hline \multirow{2}{*}{ Muestra } & \multirow{2}{*}{$\begin{array}{l}21 \\
G\end{array}$} & \multirow{2}{*}{$\begin{array}{l}50 \\
\mathrm{G}\end{array}$} & \multirow{2}{*}{$\begin{array}{l}50 \\
\text { EA }\end{array}$} & \multirow{2}{*}{$\begin{array}{l}65 \\
G\end{array}$} & \multirow{2}{*}{$\begin{array}{l}65 \\
\text { EA }\end{array}$} & \multirow{2}{*}{$\begin{array}{c}100 \\
\mathrm{G} \mathrm{c} / \mathrm{g}\end{array}$} & \multirow{2}{*}{$\begin{array}{c}100 \\
\mathrm{G} \mathrm{s} / \mathrm{g}\end{array}$} & \multirow{2}{*}{$\begin{array}{c}100 \\
\mathrm{E}\end{array}$} & \multirow{2}{*}{$\begin{array}{l}100 \\
\text { EA }\end{array}$} & \multirow{2}{*}{$\begin{array}{c}360 \\
\mathrm{G}\end{array}$} & \multirow{2}{*}{$\begin{array}{c}360 \\
\mathrm{E}\end{array}$} & \multirow{2}{*}{$\begin{array}{c}900 \\
\mathrm{E}\end{array}$} & \multicolumn{3}{|c|}{ No. Máximo } \\
\hline & & & & & & & & & & & & & $\mathrm{G}$ & $\mathrm{E}$ & EA \\
\hline $\mathrm{A} 1$ & 0 & 21 & 0 & 89 & 0 & 73 & 0 & 27 & 0 & 0 & 20 & 8 & 100 & 27 & 0 \\
\hline $\mathrm{A} 2$ & 0 & 33 & 0 & 78 & 1 & 86 & 0 & 28 & 1 & 0 & 25 & 4 & 114 & 29 & 1 \\
\hline A3 & 11 & 23 & 0 & 23 & 7 & 0 & 17 & 13 & 13 & 0 & 11 & 9 & 30 & 13 & 13 \\
\hline A4 & 9 & 13 & 0 & 4 & 12 & 0 & 5 & 15 & 15 & 0 & 18 & 7 & 20 & 18 & 18 \\
\hline A5 & 5 & 10 & 0 & 9 & 2 & 1 & 2 & 7 & 2 & 0 & 9 & 4 & 11 & 9 & 2 \\
\hline$\overline{\mathrm{X}}$ & 5 & 20 & 0 & 41 & 4.4 & 32 & 4.8 & 18 & 6.2 & 0 & 16.6 & 6.4 & 55 & 19 & 6.8 \\
\hline B1 & 0 & 16 & 0 & 10 & 2 & 0 & 9 & 8 & 8 & 0 & 10 & 5 & 17 & 10 & 8 \\
\hline B2 & 2 & 4 & 0 & 4 & 0 & 0 & 0 & 4 & 4 & 0 & 1 & 0 & 4 & 4 & 4 \\
\hline B3 & 8 & 29 & 0 & 68 & 5 & 0 & 13 & 6 & 6 & 0 & 14 & 4 & 73 & 14 & 6 \\
\hline B4 & 22 & 103 & 9 & 18 & 151 & 1 & 29 & 120 & 120 & 0 & 57 & 5 & 169 & 151 & 151 \\
\hline B5 & 2 & 49 & 0 & 15 & 39 & 24 & 2 & 26 & 26 & 0 & 20 & 0 & 54 & 39 & 39 \\
\hline$\overline{\mathrm{X}}$ & 6.8 & 40.2 & 1.8 & 23 & 39.4 & 5 & 10.6 & 32.8 & 32.8 & 0 & 20.4 & 2.8 & 63 & 44 & 41.6 \\
\hline $\mathrm{C} 1$ & 0 & 24 & 0 & 18 & 0 & 0 & 24 & 3 & 2 & 0 & 2 & 2 & 24 & 3 & 3 \\
\hline $\mathrm{C} 2$ & 3 & 6 & 0 & 8 & 0 & 1 & 8 & 0 & 0 & 8 & 0 & 6 & 9 & 6 & 0 \\
\hline $\mathrm{C} 3$ & 0 & 0 & 0 & 0 & 0 & 0 & 0 & 0 & 0 & 0 & 0 & 0 & 0 & 0 & 0 \\
\hline $\mathrm{C} 4$ & 0 & 4 & 0 & 5 & 0 & 0 & 4 & 5 & 5 & 0 & 8 & 4 & 9 & 5 & 5 \\
\hline C5 & 9 & 6 & 0 & 7 & 2 & 2 & 2 & 4 & 2 & 0 & 6 & 3 & 9 & 4 & 2 \\
\hline $\bar{X}$ & 2.4 & 8 & 0 & 8 & 0.4 & 0.6 & 7.6 & 2.4 & 1.8 & 1.6 & 3.2 & 3 & 10 & 3.6 & 2 \\
\hline D1 & 81 & 227 & 0 & 62 & 238 & 0 & 58 & 263 & 263 & 0 & 43 & 4 & 300 & 263 & 263 \\
\hline D2 & 3 & 131 & 0 & 166 & 18 & 24 & 70 & 124 & 18 & 0 & 18 & 6 & 184 & 142 & 18 \\
\hline D3 & 53 & 58 & 0 & 44 & 15 & 9 & 20 & 61 & 15 & 0 & 28 & 3 & 61 & 61 & 15 \\
\hline D4 & 66 & 66 & 0 & 119 & 2 & 44 & 43 & 66 & 27 & 0 & 27 & 9 & 153 & 66 & 27 \\
\hline D5 & 33 & 134 & 0 & 99 & 98 & 71 & 0 & 82 & 15 & 0 & 15 & 7 & 197 & 98 & 98 \\
\hline$\overline{\mathrm{X}}$ & 47.2 & 123.2 & 0 & 98 & 74.2 & 29.6 & 38.2 & 119.2 & 67.6 & 0 & 26.2 & 5.8 & 179 & 126 & 84.2 \\
\hline
\end{tabular}

Resultados a los 21, 50, 65, 100, 360 y 900 días después de inducir germinación en 20 muestras de suelo recolectadas en las cuatro áreas de estudio. $\overline{\mathrm{X}}$, promedio por zona de estudio. A, Tequexquinahuac; B, San Pablo Ixáyoc; C, Tláloc; D, Santa Catarina del Monte. G, número de gametófitos; $\mathbf{G} \mathbf{~ c / g}$, número de gametófitos con gametangios (anteridios y arquegonios); G s/g, número de gametófitos sin gametangios; $\mathbf{E}$, número de esporófitos; EA, número de esporófitos de origen apogámico.

Results on 21, 50, 65, 100 and 900 days after germination induction in 20 soil samples collected in four study sites. $\bar{X}$, average for study sites. A, Tequexquinahuac; B, San Pablo Ixayoc; C, Tlaloc; D, Santa Catarina del Monte. G, number of gametophytes; G c/g, number of gametophytes with gametangia (antheridia and archegonia); $\mathbf{G} \mathbf{~ s / g}$, number of gametophytes without gametangia; E, number of sporophytes; EA, number of apogamic sporophytes. 
CUADRO 2

Análisis de varianza de germinación de esporas entre zonas de estudio

TABLE 2

Analysis of variance of spore germination between study sites

\begin{tabular}{lccccc}
\multicolumn{1}{c}{ Fuente } & $\begin{array}{c}\text { Grados de } \\
\text { libertad }\end{array}$ & Variación & $\begin{array}{c}\text { Cuadrados } \\
\text { medios }\end{array}$ & F calculada & $\begin{array}{c}\text { Nivel de } \\
\text { probabilidad }\end{array}$ \\
Factor & 3 & 6811.75 & 2270.583 & 8.71 & $0.001178^{*}$ \\
Error & 16 & 4172.8 & 260.8 & & \\
Total (ajustado) & 19 & 10984.55 & & \\
Total & 20 & & &
\end{tabular}

*Nivel de significación de $\alpha=0.05$

Análisis de varianza de germinación de esporas considerando el número de gametófitos de 21 días, en 20 muestras de suelo recolectadas en cuatro zonas de estudio: Tequexquinahuac, San Pablo Ixáyoc, Tláloc y Santa Catarina del Monte.

Analysis of variance for spore germination considering gametophyte number at 21 days, in 20 soil samples from four study sites: Tequexquinahuac, San Pablo Ixayoc, Tlaloc and Santa Catarina del Monte.

\section{CUADRO 3}

Análisis de varianza de esporófitos entre zonas de estudio

TABLE 3

Analysis of variance of sporophytes between study sites

\begin{tabular}{|c|c|c|c|}
\hline \multicolumn{4}{|c|}{ Prueba de Kruskal-Wallis para la hipótesis nula de medianas iguales } \\
\hline Método & Grados de Libertad & Ji-cuadrada $(\mathrm{H})$ & Nivel de probabilidad \\
\hline Para tratamiento no corregido & 3 & 9.254286 & 0.026094 \\
\hline Para tratamiento corregido & 3 & 9.578365 & 0.022512 \\
\hline
\end{tabular}

Nivel de significación de $\alpha=0.05$

Análisis de varianza del número de esporófitos apogámicos, luego de 65 días después de su germinación, obtenidos de 20 muestras de suelo recolectadas en cuatro zonas de estudio: Tequexquinahuac, San Pablo Ixáyoc, Tláloc y Santa Catarina del Monte.

Analysis of variance of apogamic sporophyte number, 65 days after germination, obtained from 20 soil samples in four study sites: Tequexquinahuac, San Pablo Ixayoc, Tlaloc and Santa Catarina del Monte.

evaluación entre el cambio de generaciones, de gametófitos de 50 días a esporófitos de origen apogámico entre áreas, arrojó diferencias significativas entre los distintos sitios $(\mathrm{p}<0.05)$, pero no así entre el número de gametófitos con el de esporófitos ( $\mathrm{p}>0.05$ ) (Cuadro 5).

En la Fig. 1 se observaron las variables con mayor correlación entre las variables ambientales y los ejes de ordenación, las cuales fueron materia orgánica (0.758), textura (-632), color
(-0.550), luz (-0.405), gametófitos $(-0.262)$ y esporófitos (-0.226). En la muestra C2 se presentaron valores relativamente altos de materia orgánica, mientras que el resto de las muestras de este grupo presentaron valores más bajos. Por otro lado, las muestras de los grupos B y D revelaron niveles relativamente altos de alteración, número de esporófitos y de gametófitos. Por su parte, las muestras del grupo A representaron niveles relativamente bajos de 
CUADRO 4

Análisis de varianza de dos factores: zonas y gametófitos

TABLE 4

Two-way analysis of variance: sites and gametophytes

\begin{tabular}{lcccc}
\multicolumn{1}{c}{ Variación } & Grados de libertad & Cuadrados medios & F calculada & F tablas (0.95) \\
Áreas=4 0003.4 & 3 & 13334.4667 & 14.53 & 2.9 \\
Días (gametófitos)=10 562.5 & 1 & 10562.5 & 11.51 & 4.15 \\
Interacción=7 307.3 & 3 & 2435.76667 & 2.65 & 2.9 \\
Subtotal=57 873.2 & 7 & 8267.6 & 9.01 & \\
Aleatoria o residual=29 362.4 & 32 & 9.17575 & & \\
Total=87 235.6 & 39 & &
\end{tabular}

Análisis de varianza de dos vías: velocidad de germinación al considerar el número de gametófitos de 21 y 50 días después de inducir su germinación en 20 muestras de suelo recolectadas en cuatro zonas de estudio: Tequexquinahuac, San Pablo Ixáyoc, Tláloc y Santa Catarina del Monte.

Two-way analysis of variance: germination velocity taking into account 21 and 50 days gametophytes number after germination from 20 soil samples collected in four study sites: Tequexquinahuac, San Pablo Ixayoc, Tlaloc and Santa Catarina del Monte.

CUADRO 5

Análisis de varianza de dos factores: zonas y esporófitos

TABLE 5

Two-way analysis of variance: sites and sporophytes

\begin{tabular}{lcccc}
\multicolumn{1}{c}{ Variación } & $\begin{array}{c}\text { Grados de } \\
\text { libertad }\end{array}$ & Cuadrados medios & F calculada & F tablas $(0.95)$ \\
Áreas=54 937.075 & 3 & 18312.3583 & 7.22 & 2.9 \\
Días (gametófitos-esporófitos)=3 330.625 & 1 & 3330.625 & 1.31 & 4.15 \\
Interacción=3 426.275 & 3 & 1142.09167 & 0.45 & 2.9 \\
Subtotal=61 693.975 & 7 & 8813.425 & 3.48 & \\
Aleatoria o residual=81 156 & 32 & 2536.125 & & \\
Total=142 849.975 & 39 & & &
\end{tabular}

Nivel de significación de $\alpha=0.05$

Análisis de varianza de dos vías: número de gametofitos de 50 días y número de esporófitos apogámicos de 65 días después de inducir su germinación en 20 muestras de suelo recolectadas en cuatro áreas de estudio: Tequexquinahuac, San Pablo Ixáyoc, Tláloc y Santa Catarina del Monte.

Two-way analysis of variance: number of 50 days gametophytes and 65 days apogamic sporophytes after germination from 20 soil samples collected in four study sites: Tequexquinahuac, San Pablo Ixayoc, Tlaloc and Santa Catarina del Monte. 


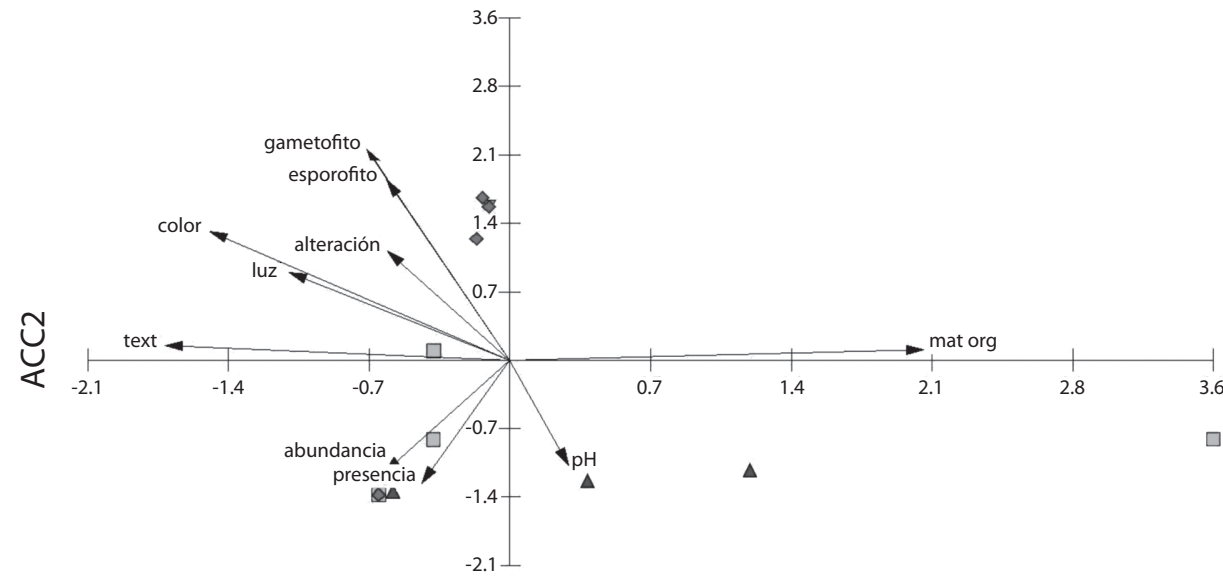

Escala del vector: 2.76

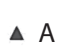

ACC1

$\Delta \mathrm{A}$

$\nabla \mathrm{B}$

$\square C$

$\diamond \mathrm{D}$

Fig. 1. Gráfico compuesto (dos vías) que muestra simultáneamente el espacio de ordenación definido por los dos primeros ejes extraídos por un Análisis de Correlación Canónica en la ordenación simultánea de la matriz de presencia-ausencia de 32 especies de helechos y licofitas y la matriz ambiental de 20 muestras dentro en cuatro localidades de la zona de estudio. Los vectores representan las variables medioambientales más importantes en la determinación de la ordenación de las comunidades de helechos. Los símbolos indican las cuatro localidades muestreadas: $\mathbf{\Delta}$ A, Tequexquinahuac; $\nabla$ B, San Pablo Ixáyoc; C C Tláloc; $\bullet$ D, Santa Catarina del Monte. Las muestras del sitio B se encuentran enmascaradas por las del sitio D.

Fig.1. Compound graph (bi-plot) from a Canonical Correspondence Analysis showing the jointly ordination of 20 samples defined by presence-absence of 32 ferns and lycophytes species restricted be the environmental variables in the study area. The vectors represent the environmental variables most important variables restricting the ordination of the ferns community. The symbols indicate the four sampled sites. $\boldsymbol{\Delta}$ A, Tequexquinahuac; $\boldsymbol{\nabla}$ B, San Pablo Ixayoc; $\mathbf{\square}$ C, Tláloc; D, Santa Catarina del Monte. Samples from site B are masked by those from site D.

alteración, pH más básico y relativamente baja incidencia de luz.

El número de gametófitos y esporófitos al igual que el grado de alteración fue significativo para las localidades $\mathrm{C}$ y $\mathrm{D}$, además, la presencia y número de helechos se relacionó de forma significativa en las localidades A, C y D.

\section{DISCUSIÓN}

El número de esporófitos de origen apogámico fue superior en las muestras de suelo recolectadas en las áreas alteradas por erosión, en Santa Catarina del Monte y en áreas perturbadas por incendios recientes en San Pablo Ixáyoc. Mickel \& Smith (2004) mencionan que Cheilanthes presenta apogamia en muchas especies basándose en el número de esporas por esporangio. Sin embargo hasta ahora no se ha citado este tipo de estrategia reproductiva en estas condiciones medioambientales y para las especies que se estudiaron. En contraste, en las muestras de suelo de zonas con baja alteración, hubo menor número de esporófitos y éstos probablemente fueron producto de reproducción sexual por la presencia de anteridios y arquegonios.

En zonas no alteradas o con baja alteración como las que existen en San Pablo, en los bosques cultivados de Tequexquinahuac y en el Tláloc, hay mayor número de especies perennes y mayor cobertura vegetal; la exposición de la ladera es menor y el grado de humedad mayor, características propicias para el crecimiento 
y desarrollo de helechos en todo el año. Los gametófitos recolectados que provienen de estas zonas formaron anteridios y arquegonios, produciendo más tarde esporófitos de origen sexual de Adiantum, Asplenium, Cheilanthes y Cystopteris (Cuadro 6).

En lugares de moderada a muy alterados, el desmonte, incendios o erosión propician la disminución de la cobertura vegetal o formación de "espacios vacíos", que provoca mayor entrada de luz, que afecta de forma directa a las esporas depositadas en la superficie del suelo. El viento esparce no sólo esporas sino también partículas de suelo y materia orgánica, provocando degradación y afectando las condiciones de sombra y humedad. El suelo de áreas alteradas funciona efectivamente como un depósito natural de esporas, sin embargo no todas tienen la capacidad de permanecer viables hasta que inicien las lluvias. Por ejemplo en Cheilanthes marginata y $C$. bonariensis solo se presenta apogamia en estas condiciones, cuando vive en sitios no alterados la formación de esporofitos se lleva a cabo por vía sexual.

Por otro lado, Vázquez et al. (1997) describen como un banco de semillas a aquel suelo que "está formado por las semillas viables no germinadas presentes en éste, ya sea enterradas, depositadas en la superficie o mezcladas en la capa de hojarasca y humus. Frecuentemente hay gran cantidad de semillas latentes en la mayoría de los suelos, su número depende de factores como la historia, la diversidad y la dinámica de la vegetación que cubre cada suelo", de tal manera que si extrapolamos esta descripción podemos decir que el suelo es un resguardo natural que las esporas tienen (banco de esporas) hasta que lleguen las condiciones propicias para su germinación y desarrollo, que para climas templados es la temporada de lluvias entre junio y septiembre.

Las montañas de la Sierra Nevada permanecen ligeramente húmedas aun en la temporada de seca gracias a los manantiales, pequeños riachuelos e infiltración del agua de lluvia; en cambio en áreas erosionadas la humedad es escasa y por tanto, las esporas forman parte del suelo prácticamente la mitad del año. En este sentido se puede explicar la diferencia del número de gametófitos en las muestras de suelo recolectadas en áreas de baja alteración, como el Tláloc, en comparación con el número de individuos que germinaron en suelo de Santa Catarina del Monte. Si bien las esporas son llevadas por el viento, la mayoría queda a poca distancia del esporófito que les dio origen y podrían permanecer viables hasta que inicien las lluvias.

En San Pablo y Tláloc es probable que las esporas inicien su germinación en cuanto llegan al suelo húmedo, eso explicaría el por que sólo se encontraron gametófitos en los meses

CUADRO 6

Gametófitos recolectados en zonas de estudio

TABLE 6

Gametophytes collected in study sites

\begin{tabular}{lcccc}
\multicolumn{1}{c}{ Muestra } & G c/g (7 días) & G c/g (180 días) & E (180 días) & Género \\
Tepechichilco & 23 & 8 & 15 & Asplenium, Cheilanthes, Cystopteris \\
Cañada Atlapulco & 36 & 28 & 8 & Adiantum, Asplenium, Cheilanthes, Cystopteris
\end{tabular}

Resultados a los 7 y 180 días después de haber recolectado gametófitos silvestres localizados dentro de la zona de estudio. Tepechichilco, San Pablo Ixáyoc y Atlapulco, Tláloc. G c/g, número de gametófitos con gametangios (anteridios y arquegonios) E, número de esporófitos.

Results of wild gametophytes after 7 and 180 days after their collection from the study sites. Tepechichilco, San Pablo Ixayoc and Atlapulco, Tlaloc. G c/g, number of gametophytes with gametangia (antheridia and archegonia); E, number of sporophytes. 
de seca en las cañadas de San Pablo y el Tláloc, por lo que en estos suelos no hay banco de esporas. En cambio en parches de vegetación primaria, rodeados de áreas erosionadas y en lugares con alto grado de degradación no fue posible observar helechos en generación gametofítica, pero si un mayor número de esporas viables en el suelo.

Las esporas germinan hasta que inician las lluvias, así que durante el tiempo de seca en suelo de áreas alteradas, de baja humedad, erosionadas y expuestas por varias horas a la luz debido a la ausencia de cobertura vegetal, sitio que funciona como depósito de esporas, que aumentan su número conforme avanza el tiempo. Se ha observado que al inducir la germinación de esporas en muestras de suelo que han sido recolectadas dos años atrás, aún se pueden obtener helechos del género Cheilanthes, lo cual coincide con lo que Dyer (1994) sugiere para las esporas de otros helechos que llevan más de dos años de almacenamiento en el suelo. Esto sugiere que quizá una ventaja adaptativa de Cheilanthes y Pellaea ternifolia subsp. ternifolia sea la capacidad de sus esporas a permanecer viables en el suelo por largos periodos de tiempo.

Page (1979) señaló que las especies de Cheilanthes con frecuencia crecen en suelo firme y duro, sobre todo en rocas ácidas de origen volcánico y sugiere que es debido a que sus hojas tienen una considerable resistencia a la desecación, por lo que se pueden desarrollar en zonas semiáridas. Tequexquinahuac y Santa Catarina, sin formar parte de zonas semiáridas, presentan características similares debido a la alteración y degradación a la que han sido sometidas, por ende la presencia y abundancia de $C$. bonariensis, C. marginata y Pellaea ternifolia subsp. ternifolia es posible tanto en estos ecosistemas como en las muestras de suelo. Como Cheilanthes y P. ternifolia subsp. ternifolia en la generación esporofítica cuentan con hojas resistentes a la desecación, e incluso algunas especies tienen abundantes escamas que reducen la pérdida de agua, también pueden sobrevivir en condiciones de aridez y llegar a producir esporas, que en los meses de lluvia germinarán para dar inicio a la fase gametofítica.

Además, las esporas de Cheilanthes bonariensis y $C$. marginata forman abundantes gametófitos y esporófitos antes que cualquier otra especie, pero son de origen apogámico (Cuadro 1). Windham \& Rabe (1993) mencionan que $C$. bonariensis es apogámico para Norte América, en el caso de las plantas de la zona de estudio esta especie se comporta apogámico sólo en sitios alterados. Los resultados indican que en condiciones de laboratorio el desarrollo de un helecho es precoz, si en lugar de desarrollar gametangios pasa directamente a la generación de esporófito, sin embargo el ciclo no se completa hasta después de dos años, tiempo dos veces mayor que el requerido para un helecho que germina en un área alterada. Los esporófitos que se desarrollan en áreas alteradas alcanzan la madurez y completan su ciclo aproximadamente en un año, por el contrario en laboratorio pasaron dos años sin que existan más cambios que su crecimiento.

Una situación diferente se presenta para Pellaea ternifolia subsp. ternifolia (Cuadro 7), si bien hay coincidencia con Cheilanthes bonariensis y $C$. marginata, en que son las especies que tienen esporas con mayor tiempo de viabilidad, aun cuando permanecen por meses en el suelo y comparten espacio en áreas de alteración media a severa, después de germinar se observan las diferencias; el desarrollo del gametófito es menos acelerado hasta formar gametangios después de tres meses de haber germinado, los esporofitos son de origen sexual, no obstante Tryon (1957) menciona que son de origen apogámico. A los 400 días de edad las plantas ya tienen esporangios aunque su talla no exceda los $15 \mathrm{~cm}$, situación que también los hace exitosos en este tipo de ecosistemas debido a la terminación temprana de su ciclo de vida.

Los resultados indican que las áreas alteradas por erosión e incendios, son el medio propicio para que individuos de Cheilanthes, principalmente $C$. bonariensis y $C$. marginata y en menor proporción Pellaea ternifolia subsp. ternifolia, colonicen espacios vacíos, ya 
CUADRO 7

Estrategias reproductivas en áreas alteradas

TABLE 7

Reproductive strategies in disturbed areas

Pellaea ternifolia subsp. ternifolia

Baja mortalidad de gametófitos

Resiste competencia por alta densidad hasta formar esporófitos

Forman gametangios

Precocidad para formar la primera hoja del esporófito

Los esporófitos son de origen sexual

Alta mortalidad de esporófitos cuando apenas se ha formado la primera hoja

Los esporófitos tienen crecimiento lento, casi dos años su talla es de $10 \mathrm{~cm}$.

Los esporangios se observan después de 400 días de haber germinado
Cheilanthes

Baja mortalidad de gametófitos

Resiste competencia por alta densidad hasta formar esporófitos

No forman gametangios

Precocidad para formar la primera hoja del esporófito

Los esporófitos son apogámicos

Baja mortalidad de esporófitos cuando apenas se ha formado la primera hoja

Los esporófitos se desarrollan rápidamente, llegando a alcanzar una talla de al menos $10 \mathrm{~cm}$ en tres meses

Los esporangios se observan después de 730 días de haber germinado

Estrategias reproductivas observadas en Pellaea ternifolia subsp. ternifolia y Cheilanthes (C. bonariensis y C. marginata) en 20 muestras de suelo recolectadas en cuatros zonas de estudio: Tequexquinahuac, San Pablo Ixáyoc, Tláloc y Santa Catarina del Monte, ubicadas en la Sierra Nevada, Texcoco, Estado de México.

Reproductive strategies observed in Pellaea ternifolia subsp. ternifolia and Cheilanthes (C. bonariensis and C. marginata) in 20 soil samples collected in four study sites: Tequexquinahuac, San Pablo Ixayoc, Tlaloc and Santa Catarina del Monte, located in the Sierra Nevada, Texcoco, Mexico State.

que tienen una mayor tolerancia al sol y forman esporófitos en menor tiempo cuando son de origen apogámico o se desarrollan rápidamente, para así producir esporas que den inicio a otro ciclo de alternancia de generaciones.

En la Sierra Nevada, la alteración de los ecosistemas ha modificado drásticamente la presencia y abundancia de helechos y licofitas, ya que aumenta el número de individuos de Cheilanthes en áreas alteradas pero disminuye la riqueza de especies, especialmente de aquellas que se reproducen sexualmente. En bosques cultivados de Pinus el comportamiento en el número y la velocidad de crecimiento de gametófitos es similar al de bosques con vegetación primaria de Quercus y Abies, debido a las prácticas de manejo de suelo.

El suelo de áreas no alteradas o de baja alteración no es adecuado como resguardo de esporas de especies comunes del ecosistema, en cambio, el suelo en los que hubo desmonte, incendio o un claro proceso de erosión, es probable que sólo permanezcan viables esporas del género Cheilanthes y esporas de Pellaea ternifolia subsp. ternifolia. La apogamia es una estrategia reproductiva exitosa para helechos del género Cheilanthes en ecosistemas alterados, porque permite que la alternancia de generaciones se realice en menos tiempo comparado con un ecosistema de baja alteración. 
La longevidad de las esporas, su fácil propagación, el tiempo relativamente pequeño en que pasa de la fase de gametófito a la de esporófito y la resistencia a la desecación o periodo de seca de algunas especies de Pellaea y Cheilanthes, las hace potencialmente útiles para propagación, retención de suelo y restitución de cobertura vegetal en una primera etapa.

\section{AGRADECIMIENTOS}

Este trabajo formó parte de los estudios de Doctorado de la primera autora en la Universidad Autónoma Metropolitana, el cual estuvo bajo la tutoría de los coautores. Se agradece la beca del Consejo Nacional de Ciencia y Tecnología, México número 164725. Mercedes Romero y Juan Antonio Rodríguez brindaron apoyo técnico durante el trabajo de campo. Robbin C. Moran y Noé Manuel Montaño Arias por la revisión crítica del manuscrito. A los revisores anónimos por sus sugerencias y atinados comentarios.

\section{RESUMEN}

Los helechos y licófitas tienen diversas estrategias reproductivas que las habilitan para invadir ambientes perturbados y áreas sin vegetación. En este trabajo se estudió la presencia, abundancia y estrategias reproductivas de individuos que crecen en suelos de bosques templados, en la Sierra Nevada, Estado de México. Se eligieron cuatro zonas en las que se instaló un cuadrante permanente de $200 \mathrm{~m}^{2}$ para cada una, con el fin de registrar variables edáficas, ambientales y de vegetación. La relación entre variables se pudo explorar con análisis de varianza y Análisis Canónico de Correspondencia dando como resultado tres paisajes con base en el grado de alteración ambiental: baja, moderada y severa. Fueron recolectadas cinco muestras de suelo en cada zona para inducir la germinación de helechos y licofitas, obteniendo resultados que revelan una relación positiva entre el grado de alteración y la abundancia de Cheilanthes. En paisajes con baja alteración, la riqueza de especies fue mayor comparada con paisajes de alteración severa, en la que la presencia de especies se redujo a Cheilanthes bonariensis, C. marginata y Pellaea ternifolia subsp. ternifolia. El suelo fue un banco de esporas de Cheilanthes y Pellaea ternifolia subsp. ternifolia ya que las esporas permanecieron viables por diferentes intervalos de tiempo. La apogamia fue la estrategia reproductiva común para especies de Cheilanthes en ecosistemas alterados, aunque estas especies tienen reproducción sexual en ecosistemas naturales con suficiente agua. Por otro lado, Pellaea ternifolia subsp. ternifolia solo presentó reproducción sexual. La apogamia puede estar relacionada con que la alternancia de generaciones se realice en menos tiempo, comparado con un ecosistema de baja alteración.

Palabras clave: alteración ambiental, apogamia, banco de esporas, helechos, licófitas, México.

\section{REFERENCIAS}

Bell, P.R. 1979. The contribution of the ferns to an understanding of the life cycles of vascular plants, p. 58-85. In A.F. Dyer (ed.). The experimental biology of ferns. Academic, London, England.

Cosío, C. 2001. Panorama socioeconómico y demográfico de Texcoco. Indicadores para la planeación municipal. Universidad Autónoma de Chapingo, Texcoco, México.

Department of Agriculture. 1998. Munsell soil color charts. United State Department of Agriculture, Washington D.C., EEUU.

Dyer, A.F. 1994. Natural soil spore banks -can they be used to retrieve lost ferns? Biodivers. Conserv. 3: 160-175.

Dyer, A.F. \& S. Lindsay. 1992. Soil spore banks of temperate ferns. Amer. Fern J. 82: 89-122.

Farrar, D.R., C. Dassler, J.E. Watkins Jr. \& C. Skelton. 2008. Gametophyte ecology, p. 222-256. In T.A. Ranker \& C.H. (eds.). Haufler. Biology and Evolution of Ferns and Lycophytes. Cambridge, Cambridge, England.

García, E. \& W. Köeppen. 1973. Modificaciones al sistema de clasificación climática de Köeppen (para adaptarla a las condiciones de la República Mexicana). Instituto de Geografía, Universidad Nacional Autónoma de México, México D.F., México.

Instituto Nacional de Estadística, Geografía e Informática. 2000. Cuaderno Estadístico Municipal de Texcoco, Estado de México, México.

Kovach, W.L. 2004. MVSP - A MultiVariate Statistical Package for Windows, ver. 3.12. Kovach Computing Services, Pentraeth, Gales, England.

Kruckeberg, A.R. 1964. Ferns associated with ultramafic rocks in the Pacific Northwest. Am. Fern J. 54: 113-126. 
Lloyd, R.M. 1974. Reproductive biology and evolution in the pteridophyta. Ann. Missouri Bot. Gard. 61: 318-331.

Lorea, F. \& R. Riba. 1990. Guía para la recolección y preparación de ejemplares para herbario de pteridofitas. Consejo Nacional de la Flora de México, México D.F., México.

Mickel, J.T. \& A.R. Smith. 2004. The Pteridophytes of Mexico. Mem. New York Bot. Gard. 88: 1-1054.

Page, C.N. 1979. The diversity of ferns. An ecological perspective, p. 9-56. In A.F. Dyer. (ed.). The experimental biology of ferns. Academic, London, England.

Rincón-Gallardo, P.E. 1991. Análisis estructural de la comunidad de helechos terrestres en una selva húmeda en "Los Tuxtlas", Veracruz, México. Tesis de licenciatura, Universidad Nacional Autónoma de México, México D.F., México.

Rodríguez, L., L. Pacheco \& J.A. Zavala. 2008. Pteridofitas indicadoras de alteración ambiental en el bosque templado de San Jerónimo Amanalco, Texcoco, México. Rev. Biol. Trop. 56: 641-656.

Rzedowski, J. 1978. Vegetación de México. Limusa, México D.F., México.

Sheffield, E. \& P.R. Bell. 1987. Current studies of the pteridophyte life cycle. Bot. Rev. 53: 442-489.

Sheffield, E. 2008. Alternation of generations, p. 49-63. In T. Ranker \& C.H. Haufler (eds.). Biology and Evolution of ferns and Lycophytes. Cambridge, Cambridge, London.

Siebe, C., R. Jahn \& K. Stahr. 1996. Manual para la descripción y evaluación ecológica de suelos en el campo. Sociedad Mexicana de la Ciencia del Suelo, A.C. Publicación Especial 4. Chapingo, Texcoco, México.

Smith, D.L. 1979. Biochemical and physiological aspects of gametophyte differentiation and development, $\mathrm{p}$. 355-392. In A.F. Dyer (ed.). The experimental biology of ferns. Academic, London, England.
Spiegel, M.R. \& L.J. Stephens. 2002. Estadística. McGrawHill, Interamericana, México D.F., México.

Tryon, A.F. 1957. A revision of the fern genus Pellaea section Pellaea. Ann. Missouri Bot. Gard. 44: 125-193.

Tryon, R.M. 1986. The biogeography of species, with special reference to ferns. Bot. Rev. 52: 118-156.

Tuomisto, H. \& A.D. Poulsen. 1996. Influence of edaphic specialization on pteridophyte distribution in neotropical rain forests. J. Biogeography 23: 283-293.

Tuomisto, H. \& K. Ruokolainen. 1994. Distribution of pteridophyta and Melastomataceae along an edaphic gradient in an Amazonian rain forest. J. Veg. Sci. 5: 25-34.

Tuomisto, H., A.D. Poulsen \& R.C. Moran. 1998. Edaphic distribution of some species of the fern genus Adiantum in western Amazonia. Biotropica 30: 392-399.

Valdés, J. \& H. Flores. 1983. Las pteridófitas en la flora halófila y gipsófila de México. Serie Botánica. An. Inst. Biol. Univ. Nal. Autón. México 54: 173-188.

Vázquez, C., A. Orozco, M. Rojas, M.E. Sánchez \& V. Cervantes. 1997. La reproducción de las plantas: semillas y meristemos. Ciencia 157. Fondo de Cultura Económica, México D.F., México.

Walkley, A. \& I.A. Black. 1934. An examination of the Detjareff method for determining soil organic matter and propose modification of the chromic acid titration method. Soil Sci. 37: 29-38.

Wherry, E.T. 1920. The soil reactions of certain rock ferns -II. Amer. Fern J. 10: 45-52.

White, R.A. 1979. Experimental investigations of fern sporophyte development, p. 505-549. In A.F. Dyer (ed.). The experimental biology of ferns. Academic, London, England.

Windham, M.D. \& E.W. Rabe. 1993. Cheilanthes, p. 152170. In N.R. Morin (ed.). Flora of North America and North of Mexico. Oxford, Nueva York, USA. 
APÉNDICE A

Variables ambientales

APPENDIX A

Environmental variables

\begin{tabular}{|c|c|}
\hline Variable & Categoría \\
\hline Color & $\begin{array}{l}\text { 1. Gris } \\
\text { 2. Pardo } \\
\text { 3. Amarillo }\end{array}$ \\
\hline Textura & $\begin{array}{l}\text { 1. Arena } \\
\text { 2. Franca } \\
\text { 3. Limo } \\
\text { 4. Arcilla }\end{array}$ \\
\hline Capacidad de infiltración & $\mathrm{ml} / \mathrm{s}$ \\
\hline $\mathrm{pH}$ & $1-14$ \\
\hline Contenido de materia orgánica $(\%)$ & $0-100$ \\
\hline Exposición a la luz & $\begin{array}{l}\text { 1. Escasa } \\
\text { 2. Interrumpida } \\
\text { 3. Luminosa } \\
\text { 4. Muy luminosa }\end{array}$ \\
\hline Nivel de alteración & $\begin{array}{l}\text { 1. Muy bajo } \\
\text { 2. Moderado } \\
\text { 3. Alto } \\
\text { 4. Muy alto }\end{array}$ \\
\hline Gametófitos & Número total de gametófitos por muestra de suelo \\
\hline Esporófitos & Número total de esporófitos por muestra de suelo \\
\hline Apogamia & $\begin{array}{l}\text { Número de esporófitos de origen apogámico por } \\
\text { muestra de suelo }\end{array}$ \\
\hline Riqueza de especies & Número de especies presentes en cada muestra de suelo \\
\hline Abundancia & Número de individuos presentes en muestra de suelo \\
\hline
\end{tabular}

Variables y categorías utilizadas en 20 sitios de muestreo y 20 muestras de suelo obtenidas en Tequexquinahuac, San Pablo Ixáyoc, Tláloc y Santa Catarina del Monte

Registration variables in 20 sampling places and 20 soil sampling in Tequexquinahuac, San Pablo Ixayoc, Tlaloc and Santa Catarina del Monte. 
APÉNDICE B

Especies de helechos y licofitas reconocidas en campo en las zonas de estudio

\section{APPENDIX B}

Ferns and Lycophytes species recorded in the study sites

\begin{tabular}{|c|c|c|c|c|c|}
\hline Num & Especie & A & B & $\mathrm{C}$ & $\mathrm{D}$ \\
\hline 1 & Adiantum andicola Liebm. & & & $\mathrm{X}$ & \\
\hline 2 & Adiantum capillus-veneris $\mathrm{L}$. & & $\mathrm{X}$ & & \\
\hline 3 & Adiantum poiretii Wikstr. & $\mathrm{X}$ & $\mathrm{X}$ & $\mathrm{X}$ & $\mathrm{X}$ \\
\hline 4 & Argyrochosma incana (C. Presl) Windham & & $\mathrm{X}$ & & $\mathrm{X}$ \\
\hline 5 & Asplenium monanthes $\mathrm{L}$. & & $\mathrm{X}$ & $\mathrm{X}$ & \\
\hline 6 & Astrolepis crassifolia (T. Moore \& Houlston) D. M. Benham \& Windham & $\mathrm{X}$ & & & \\
\hline 7 & Athyrium bourgeaui $\mathrm{E}$. Fourn & & & $\mathrm{X}$ & \\
\hline 8 & Botrychium virginianum (L.) Sw. & & $\mathrm{X}$ & & \\
\hline 9 & Cheilanthes arizonica (Maxon) Mickel & & $\mathrm{X}$ & & \\
\hline 10 & Cheilanthes bonariensis (Willd.) Proctor & $\mathrm{X}$ & $\mathrm{X}$ & & $\mathrm{X}$ \\
\hline 11 & Cheilanthes kaulfusii Kunze & $\mathrm{X}$ & $\mathrm{X}$ & & \\
\hline 12 & Cheilanthes lendigera (Cav.) Sw. & & $\mathrm{X}$ & $\mathrm{X}$ & \\
\hline 13 & Cheilanthes marginata Kunth & $\mathrm{X}$ & $\mathrm{X}$ & $\mathrm{X}$ & $\mathrm{X}$ \\
\hline 14 & Cheilanthes marsupianthes (Fée) T. Reeves ex Mickel \& A. R. Sm. & & $\mathrm{X}$ & $\mathrm{X}$ & \\
\hline 15 & Cheilanthes membranacea (Davenp.) Maxon & $\mathrm{X}$ & & & \\
\hline 16 & Cheilanthes myriophylla Desv. & $\mathrm{X}$ & $\mathrm{X}$ & & $\mathrm{X}$ \\
\hline 17 & Cheilanthes purpusii $\mathrm{T}$. Reeves & & & & $\mathrm{X}$ \\
\hline 18 & Cheilanthes pyramidalis Fée & $\mathrm{X}$ & & & $\mathrm{X}$ \\
\hline 19 & Cystopteris fragilis (L.) Bernh. & $\mathrm{X}$ & $\mathrm{X}$ & $\mathrm{X}$ & \\
\hline 20 & Dryopteris pseudofilix-mas (Fée) Rothm & & & $\mathrm{X}$ & \\
\hline 21 & Pellaea cordifolia (Sessé \& Moç) A. R. Sm. & $\mathrm{X}$ & $\mathrm{X}$ & & \\
\hline 22 & Pellaea ovata (Desv.) Weath. & $\mathrm{X}$ & $\mathrm{X}$ & & \\
\hline 23 & Pellaea sagittata (Cav.) Link & $\mathrm{X}$ & & & \\
\hline 24 & Pellaea ternifolia (Cav.) Link subsp. ternifolia & $\mathrm{X}$ & $\mathrm{X}$ & & $\mathrm{X}$ \\
\hline 25 & Pleopeltis polylepis (Roemer ex Kunze) T. Moore & & $\mathrm{X}$ & $\mathrm{X}$ & \\
\hline 26 & Polypodium guttatum Maxon & & $\mathrm{X}$ & $\mathrm{X}$ & \\
\hline 27 & Polypodium thyssanolepis A. Braun ex Klotzsch & & $\mathrm{X}$ & $\mathrm{X}$ & \\
\hline 28 & Polystichum fournieri A. R. Sm. & $\mathrm{X}$ & & & \\
\hline 29 & Selaginella lepidophylla (Hook. \& Grev.) Spring & $\mathrm{X}$ & & & \\
\hline 30 & Selaginella sellowii Hieron & $\mathrm{X}$ & & & \\
\hline 31 & Woodsia mollis (Kaulf.) J. Sm. & $\mathrm{X}$ & $\mathrm{X}$ & $\mathrm{X}$ & $\mathrm{X}$ \\
\hline
\end{tabular}

Presencia de helechos y licofitas en las zonas de estudio: A, Tequexquinahuac; B, San Pablo Ixáyoc; C, Tláloc; D, Santa Catarina del Monte. Se ordenaron alfabéticamente y se asignó un número.

Presence of ferns and licophytes in field in the study sites: A, Tequexquinahuac; B, San Pablo Ixayoc; C, Tlaloc; D, Santa Catarina del Monte.. The order is alphabetical and assigned number. 
APÉNDICE C

Abundancia de especies de helechos en banco de esporas del suelo

\section{APPENDIX C}

Abundance of ferns species in the spore bank in soil

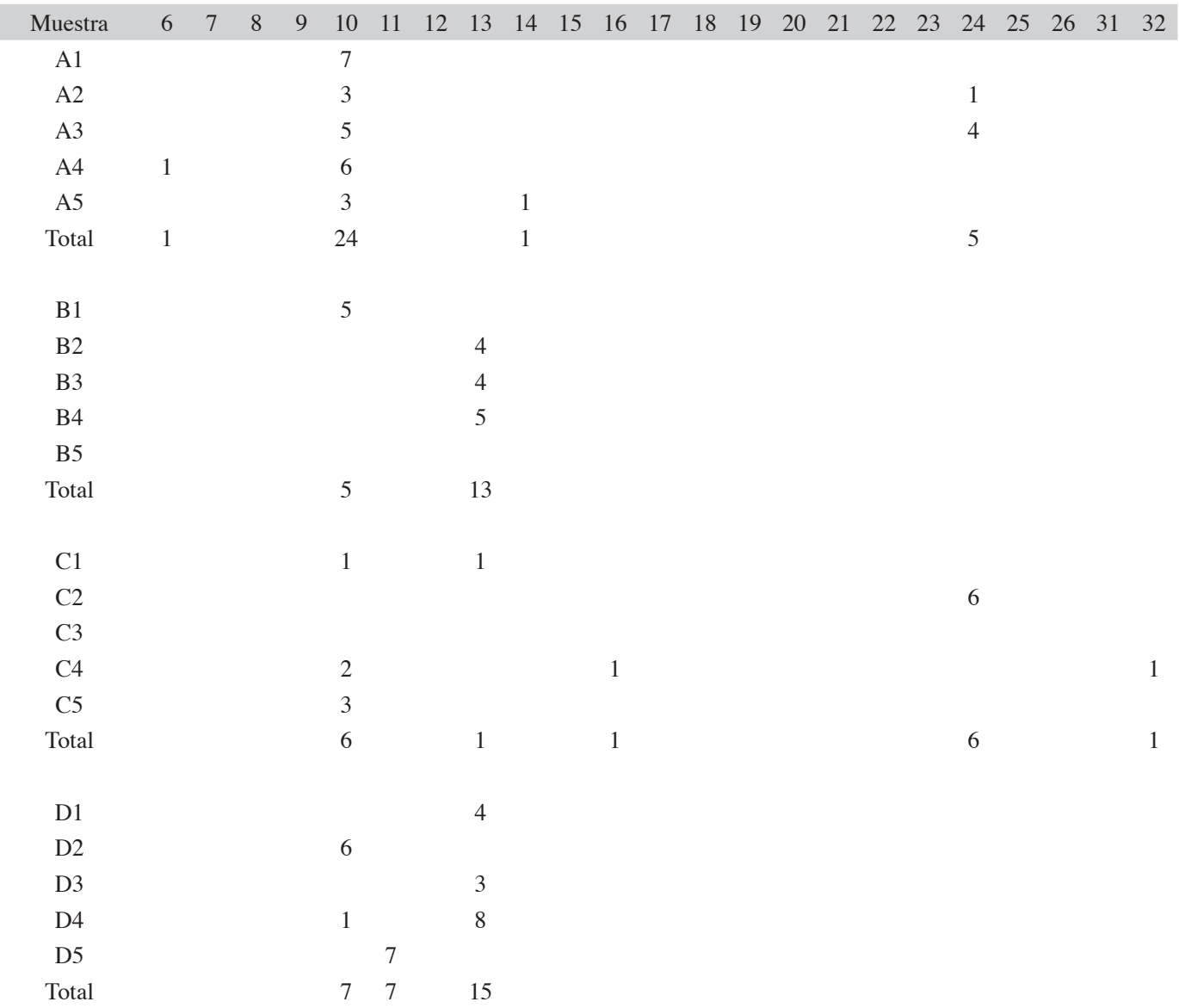

Número de individuos por especie obtenidos en 20 muestras de suelo en cuatro zonas de estudio, después de 900 días de haber inducido su germinación. El número de especie corresponde al listado del Apéndice B. Destaca por la abundancia de Cheilanthes bonariensis, C. marginata y Pellaea ternifolia subsp. ternifolia con 42, 29 y 11 individuos respectivamente. A, Tequexquinahuac; B, San Pablo Ixáyoc; C, Tláloc; D, Santa Catarina del Monte. El número 32 se refiere a Astrolepis integerrima (Hook.) D.M. Benham \& Windham, la cual no había sido recolectada en campo durante los recorridos previos.

Individuals number per species 900 days after germination in 20-sampled soil of four study sites. Species number corresponds to Appendix B. Emphasize for abundance Cheilanthes bonariensis, C. marginata and Pellaea ternifolia subsp. ternifolia with 42, 29 and 11 individuals respective. A, Tequexquinahuac; B, San Pablo Ixayoc; C, Tláloc; D, Santa Catarina del Monte. The number 32 is Astrolepis integerrima (Hook.) D.M. Benham \& Windham, which one has not reported in previous field trails. 